\title{
Oxidative stress response to menadione and cumene hydroperoxide in the opportunistic fungal pathogen Candida glabrata
}

\author{
Mayra Cuéllar-Cruz ${ }^{1,2}$, Irene Castaño', Omar Arroyo-Helguera', Alejandro De Las Peñas ${ }^{1 /+}$ \\ División de Biología Molecular, Instituto Potosino de Investigación Científica y Tecnológica, Camino a la Presa San José 2055, 78216 \\ San Luis Potosí, SLP, México ²Universidad Autónoma del Estado de Hidalgo, Instituto de Ciencias Básicas e Ingeniería, \\ Área Académica de Química, 42060 Pachuca, HGO, México
}

Candida glabrata is an opportunistic fungal pathogen that can cause severe invasive infections and can evade phagocytic cell clearance. We are interested in understanding the virulence of this fungal pathogen, in particular its oxidative stress response. Here we investigated C. glabrata, Saccharomyces cerevisiae and Candida albicans responses to two different oxidants: menadione and cumene hydroperoxide (CHP). In log-phase, in the presence of menadione, C. glabrata requires Ctalp (catalase), while in a stationary phase (SP), Ctalp is dispensable. In addition, C. glabrata is less resistant to menadione than $\mathrm{C}$. albicans in SP. The S. cerevisiae laboratory reference strain is less resistant to menadione than $\mathrm{C}$. glabrata and $\mathrm{C}$. albicans; however $\mathrm{S}$. cerevisiae clinical isolates (CIs) are more resistant than the lab reference strain. Furthermore, S. cerevisiae CIs showed an increased catalase activity. Interestingly, in SP C. glabrata and S. cerevisiae are more resistant to CHP than $\mathrm{C}$. albicans and Ctalp plays no apparent role in detoxifying this oxidant.

Key words: Candida glabrata - menadione - cumene - CTA1 - Candida albicans - Saccharomyces cerevisiae

Reactive oxygen species (ROS), including superoxide ion, hydrogen peroxide and hydroxyl radicals, are normal by-products of aerobic respiration. ROS can damage all biomolecules, but cells have developed enzymatic (catalases, superoxide dismutases and peroxidases) and nonenzymatic (glutathione and thioredoxin) mechanisms for keeping ROS levels low. Phagocytic cells, which are the first line of defence against fungal infections, generate ROS in order to eliminate invading pathogens (Mansour \& Levitz 2002). Interestingly, pathogens have co-opted these well-conserved antioxidant mechanisms to evade phagocyte defences, thus survival and persistence are ensured (Thorpe et al. 2004, Temple et al. 2005).

Candida glabrata is an opportunistic fungal pathogen that can cause severe invasive infections and recent surveys show that $C$. glabrata is one of the most frequently isolated species in hospital acquired disseminated infections (Trick et al. 2002). C. glabrata virulence factors have only recently begun to be identified (Kaur et al. 2005). C. glabrata has a well-defined oxidative stress response and we have previously found that $C$. glabrata can withstand very high concentrations of $\mathrm{H}_{2} \mathrm{O}_{2}$ relative to that of Saccharomyces cerevisiae and even Candida albicans (Cuéllar-Cruz et al. 2008). Resistance of cells to $\mathrm{H}_{2} \mathrm{O}_{2}$ in stationary phase (SP) is dependent on the

Financial support: MCC (163140) and ADLP (CB-2005-48279) are fellows from CONACyT.

+Corresponding author: cano@ipicyt.edu.mx

Received 10 August 2008

Accepted 16 April 2009 concerted role of Yap1, Skn7 and Msn4, well-conserved stress-related transcription factors. In log-phase cells (LP) C. glabrata adapts to high levels of $\mathrm{H}_{2} \mathrm{O}_{2}$ and this adaptive response is dependent on Yap1 and Skn7 and partially on Msn2 and Msn4. The single catalase gene Ctal is absolutely required for resistance to $\mathrm{H}_{2} \mathrm{O}_{2}$ in vitro, however in a mouse model of systemic infection the catalase is dispensable. A strain lacking Ctal showed no effect on virulence (Cuéllar-Cruz et al. 2008).

In this report, to better understand the oxidative stress response in C. glabrata, we screened the resistance of $C$. glabrata to two different oxidants: menadione and cumene hydroperoxide (CHP). We asked whether C.g.Ctalp is important for this response. We compared the resistance to these oxidants with the distantly related fungal opportunistic pathogen C. albicans and the nonpathogenic and closely related yeast $S$. cerevisiae. For $S$. cerevisiae we used a laboratory reference strain and two clinical isolates (CIs). In addition, we determined catalase activity in these strains.

\section{MATERIALS AND METHODS}

Strains - All strains used in this study are summarised in Table.

Media - Yeast media were prepared as described (Ausubel et al. 2001) and 2\% agar was added for plates. YPD media contained yeast extract $(10 \mathrm{~g} / \mathrm{L})$ and peptone ( $20 \mathrm{~g} / \mathrm{L})$, supplemented with $2 \%$ glucose.

Menadione and CHP sensitivity assays - All the starting overnight cultures of $C$. glabrata, $C$. albicans and $S$. cerevisiae were grown in YPD for $36 \mathrm{~h}$ at $30^{\circ} \mathrm{C}$. Menadione and CHP (88\%) were purchased from SigmaAldrich. All liquid cultures and plates were incubated at $30^{\circ} \mathrm{C}$. For sensitivity assays for LP cells overnight cultures were diluted in fresh rich media, YPD, in such a 
way that cells went through seven doublings (D) to reach $\mathrm{OD}_{600 \mathrm{~nm}} 0.5$. The cultures were divided, exposed to different menadione or CHP concentrations and incubated with shaking for $3 \mathrm{~h}$. After the treatment the oxidant was removed and the cultures were adjusted when needed to O.D. ${ }_{600 \mathrm{~nm}} 0.5$, serially diluted in 96 -well plates and each dilution was spotted onto YPD plates and incubated at $30^{\circ} \mathrm{C}$. Each dilution had the same amount of cells.

For SP cells, $36 \mathrm{~h}$ cultures were diluted to O.D. ${ }_{600 \mathrm{~mm}}$ 0.5 with spent media from the same culture. The cells were divided into aliquots and treated with menadione or CHP at different concentrations for $3 \mathrm{~h}$ at $30^{\circ} \mathrm{C}$. After the treatment, the oxidant was removed and the cultures remained at the same O.D. ${ }_{600 \mathrm{~nm}} 0.5$. The cultures were diluted in 96-well plates and spotted onto YPD plates.

All manipulations for these assays were performed in our $30^{\circ} \mathrm{C}$ temperature-controlled room to prevent abrupt changes in temperature because it has been reported that cold shock has an impact on oxidative stress resistance in $S$. cerevisiae. The experiments were repeated three times.

Preparation of cell-free extracts and catalase activity assay - The cells were grown for $48 \mathrm{~h}$ in YPD containing $2 \%$ glucose and were harvested by centrifugation at 800 $g$ for $10 \mathrm{~min}$. Cells were washed twice with distilled water and resuspended in lysis buffer [potassium phosphate (pH 7.0) supplemented with SIGMAFAST protease inhibitor from SIGMA] and $0.5 \mathrm{~g}$ of glass beads (SIGMA). Cells were frozen and thawed two times and the mixture was vortexed 4-6 times with 1 min cooling on ice. The lysate was centrifuged for $20 \mathrm{~min}$ at $4^{\circ} \mathrm{C}$ to remove cell debris and glass beads. The supernatant was used for enzymatic activity. Protein quantification was determined by Bradford assay (Bradford 1976). Bovine serum albumin from Sigma was used as standard. Catalase activity was determined in cell-free extracts by a spectrophotometric method that measures the breakdown of $\mathrm{H}_{2} \mathrm{O}_{2}$ by catalase (Aebi 1984). Assays were performed four times. The catalase activity was normalised to total protein from the lysate and expressed as units per mg of protein. One unit is defined as the amount of catalase required for degradation of $1.0 \mu \mathrm{mol} \mathrm{H} \mathrm{H}_{2} \mathrm{O}_{2}$ per min.

\section{RESULTS}

C. glabrata resistance to menadione - In order to understand the oxidative stress response in C. glabrata, we decided to assess its resistance in LP cells to menadione, a cytotoxic quinone that generates superoxide. The cells were grown and exposed to menadione (Fig. 1). C. glabrata BG14 (wt, reference strain) grows in up to $0.2 \mathrm{mM}$ menadione. However, the ctal $\Delta$ strain, which lacks the single catalase gene, showed reduced resistance to menadione (Fig. 1A). We then assessed whether C. glabrata cells are more resistant to menadione in SP (Fig. 1). Both strains BG14 and ctal $\Delta$ exhibited increased resistance to menadione to the same extent (Fig. 1B).

C. albicans, S. cerevisiae and C. glabrata resistance to menadione in SP - Once we had established C. glabrata resistance to menadione in SP and that Cta1 was dispensable, we decided to compare its resistance with a $C$. albicans strain (an opportunistic fungal pathogen), a $S$. cerevisiae lab reference strain (non-pathogenic) and two S. cerevisiae CIs in SP. The cells were grown and exposed to menadione (Fig. 2). C. albicans, which is distantly related to C. glabrata, is more resistant to menadione than C. glabrata (Fig. 2). C. albicans can grow in up to $1.2 \mathrm{mM}$ menadione while C. glabrata can only grow in up to $0.8 \mathrm{mM}$ (Fig. 2). S. cerevisiae lab reference strain W303 showed resistance only up to $0.2 \mathrm{mM}$ menadione. However, both S. cerevisiae CIs (YJM128 and YJM336) showed increased resistance to menadione. These strains were able to grow in the presence of $0.6 \mathrm{mM}$ menadione (Fig. 2).

C. albicans, S. cerevisiae and C. glabrata resistance to $C H P$ in $S P$ - We decided to determine the resistance of these fungal strains to CHP, an organic hydroperoxide, in SP. The cells were grown and exposed to CHP (Fig. 3). Both strains, BG14 and ctal $\Delta$, were resistant to $0.4 \mathrm{mM}$ CHP (Fig. 3) and more resistant than C. albicans strain CA5, which showed sensitivity to $0.4 \mathrm{mM}$ (Fig. 3). Interestingly, the non-pathogenic S. cerevisiae strain W303 and both CI strains, YJM128 and JYM336, showed almost the same resistance at $0.4 \mathrm{mM} \mathrm{CHP}$ as $C$. glabrata. At this concentration, C. albicans strain CA5 is completely sensitive (Fig. 3).

TABLE

Strains used in this study

\begin{tabular}{|c|c|c|c|c|}
\hline & Strain & Parent & Genotype & Reference \\
\hline \multirow[t]{3}{*}{ Saccharomyces cerevisiae strain } & W303 & & $\begin{array}{l}\text { MATa ura3-1 leu2-3,112 his3-11,15 trp1-1 } \\
\text { can1-100 ade2-1 ade3::hisG }\end{array}$ & McDonald et al. (1997) \\
\hline & YJM128 & & Clinical isolate & Clemons et al. (1994) \\
\hline & YJM336 & & Clinical isolate & Clemons et al. (1994) \\
\hline Candida albicans strain & CA5 & & Clinical isolate & Laboratory collection \\
\hline \multirow[t]{3}{*}{ Candida glabrata strains } & BG2 & & Clinical isolate (strain B) & Fidel et al. (1996) \\
\hline & BG14 & BG2 & 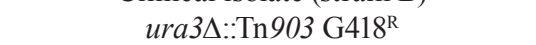 & Cormack and Falkow (1999) \\
\hline & $\begin{array}{l}\text { CGM295 } \\
\text { ctals }\end{array}$ & BG14 & 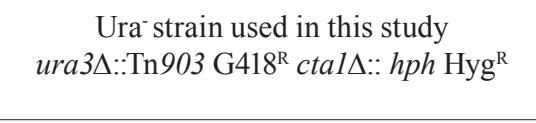 & Cuéllar-Cruz et al. (2008) \\
\hline
\end{tabular}


A

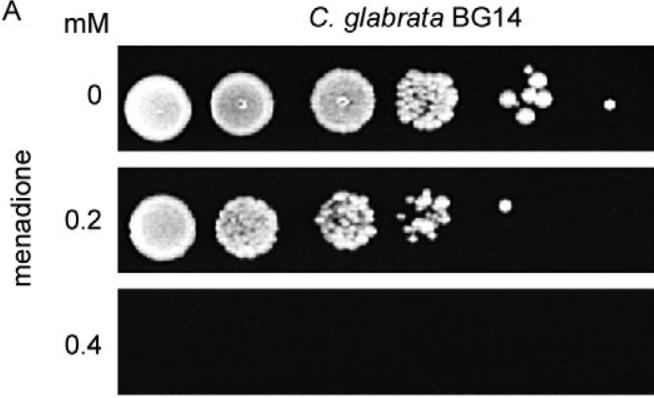

B
$\mathrm{mM}$
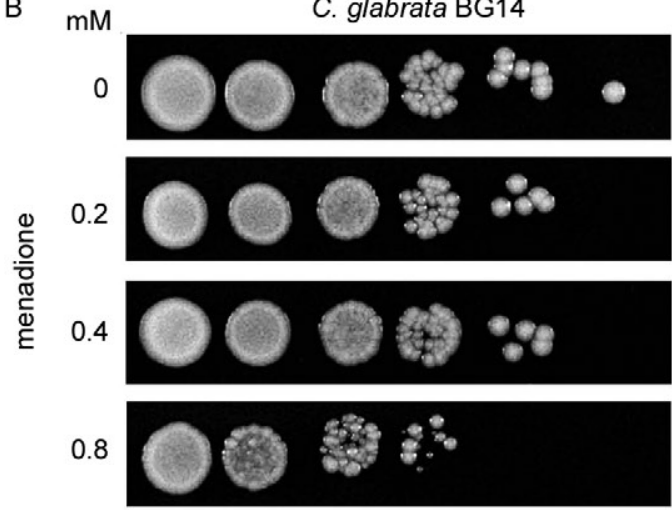

1.2

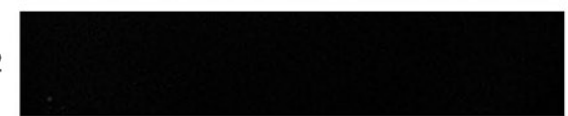

$\operatorname{cta1\Delta }$

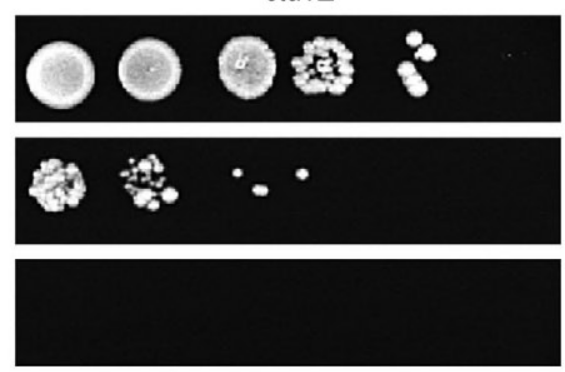

$\operatorname{cta1\Delta }$
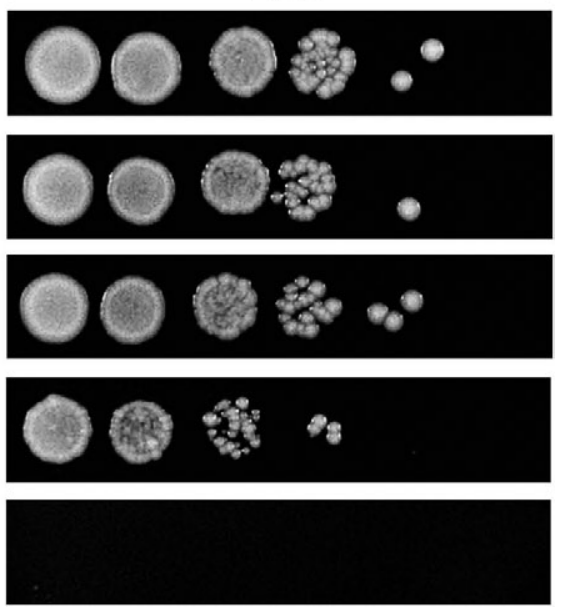

Fig. 1: Candida glabrata resistance to menadione. A: for log-phase cells LP sensitivity assay, saturated cultures of C. glabrata strains BG14 (wt) and CGM295 (ctal $\Delta$ ) were diluted into fresh media (YPD) so that cells would reach an $\mathrm{OD}_{600 \mathrm{~mm}} 0.5$ after seven doublings at $30^{\circ} \mathrm{C}$. C. glabrata BG14 and CGM295 cultures were divided and exposed to $0,0.2$ and $0.4 \mathrm{mM}$ of menadione. After the treatment, the cultures were adjusted when needed to O.D. ${ }_{600 \mathrm{~mm}} 0.5$, serially diluted and each dilution spotted into YPD plates, assuring that same amount of cells is plated. Plates were incubated at $30^{\circ} \mathrm{C}$; B: for stationary phase sensitivity assay, saturated cultures of C. glabrata strains BG14 (wt) and CGM295 (ctal $\Delta$ ) were diluted to O.D. ${ }_{600 \mathrm{~nm}} 0.5$ with spent media from the same culture. The cells were divided into aliquots and treated for $3 \mathrm{~h}$ with menadione at different concentrations: $0,0.2,0.4,0.8$ and $1.2 \mathrm{mM}$. After the treatment, the cultures remained at O.D. ${ }_{600 \mathrm{~mm}} 0.5$, oxidant was removed and cells were diluted and spotted onto YPD plates. Plates were incubated at $30^{\circ} \mathrm{C}$.

C. glabrata, C. albicans and S. cerevisiae catalase activity - We have previously shown in plate assays that C. glabrata is more resistant to $\mathrm{H}_{2} \mathrm{O}_{2}$ than C. albicans and S. cerevisiae (Cuéllar-Cruz et al. 2008). Therefore, we decided to assay catalase activity in these strains. Surprisingly, C.g.B14, C.a.CA5 and S.c.W303 showed almost no difference in catalase activity (Fig. 4). As expected, the C. glabrata strain C.g.ctal $\Delta$ that lacks catalase showed no activity. However, $S$. cerevisiae CIs showed increased catalase activity, consistent with the fact that these $S$. cerevisiae CIs are more resistant to $\mathrm{H}_{2} \mathrm{O}_{2}$ than C.a.CA5 and S.c.W303 (Cuéllar-Cruz et al. 2008) (Fig. 4).

\section{DISCUSSION}

Pathogens are able to evade oxidative killing by phagocytic cells by using the well conserved enzymatic and non-enzymatic mechanisms that keep ROS, generated naturally by aerobic respiration, at low levels (Gonzalez-Parraga et al. 2003). In this way, pathogens can survive and persist in their host. The oxidative stress response for C. glabrata has only recently been described
(Chen et al. 2007, Cuéllar-Cruz et al. 2008, Roetzer et al. 2008). C. glabrata is extremely resistant to very high levels of $\mathrm{H}_{2} \mathrm{O}_{2}$ and can evade phagocytic cell clearance (Kaur et al. 2007, Cuéllar-Cruz et al. 2008). C. glabrata has one catalase (Ctalp) that is required to respond to oxidative stress generated by $\mathrm{H}_{2} \mathrm{O}_{2}$ (Cuéllar-Cruz et al. 2008). Furthermore, the well-conserved transcription factors Yap1, Skn7, Msn2 and Msn4 coordinate, in part, the oxidative stress response in C. glabrata (Chen et al. 2007, Cuéllar-Cruz et al. 2008, Roetzer et al. 2008). In this report, we investigated the natural resistance of $C$. glabrata, C. albicans and S. cerevisiae to menadione and CHP. In addition, we measured their catalase activity in SP. Fig. 1A shows that LP cells of C.g.BG14 (wt) can resist up to $0.2 \mathrm{mM}$ menadione and that this resistance is dependent on Cta1. Menadione is a cytotoxic quinone that generates superoxide and it has been shown that exposure to menadione induces the expression of S.c. and S.p.Ctal (Nakagawa et al. 1995, Osorio et al. 2003). This result suggests that the superoxide generated by menadione is dismutated to $\mathrm{H}_{2} \mathrm{O}_{2}$, which is then reduced by the 


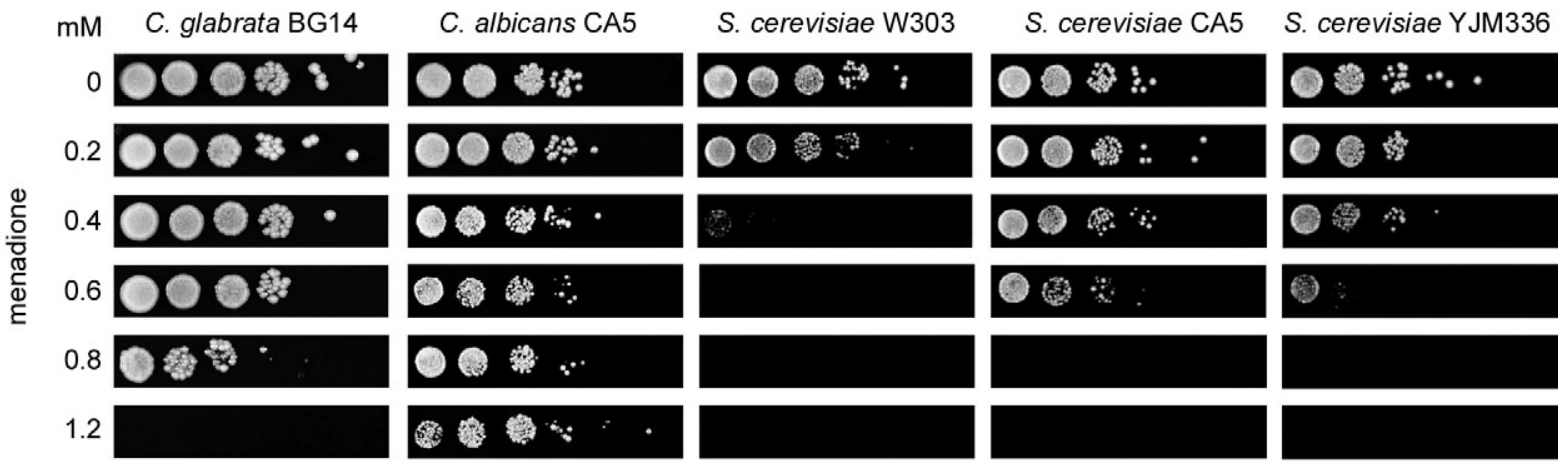

Fig. 2: Candida glabrata, Candida albicans and Saccharomyces cerevisiae stationary phase resistance to menadione. Saturated cultures of $C$. glabrata strain BG14, C. albicans strain CA5, S. cerevisiae strains W303, CIs YJM128 and YJM336 were treated as described in Fig. 1B.

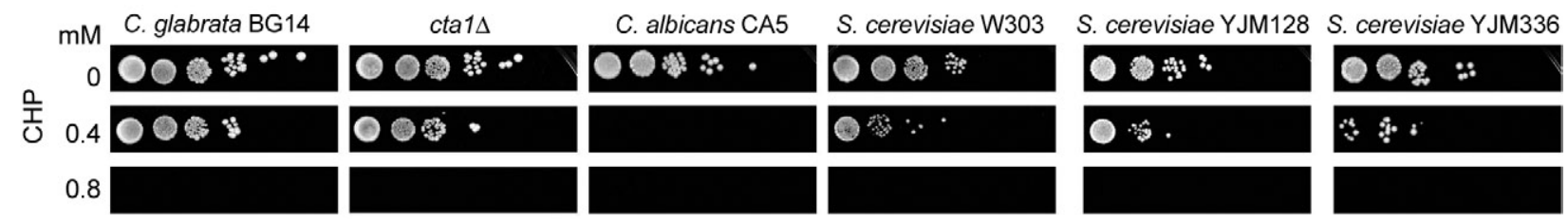

Fig. 3: Candida glabrata, Candida albicans and Saccharomyces cerevisiae stationary phase resistance to cumene hydroperoxide (CHP). Saturated cultures of C. glabrata strains BG14 and CGM295 (cta1A), C. albicans strain CA5, S. cerevisiae strains W303, CIs YJM128 and YJM336 were grown and treated as in Fig. 2, but cultures were expose to CHP at $0,0.4$ and $0.8 \mathrm{mM}$.

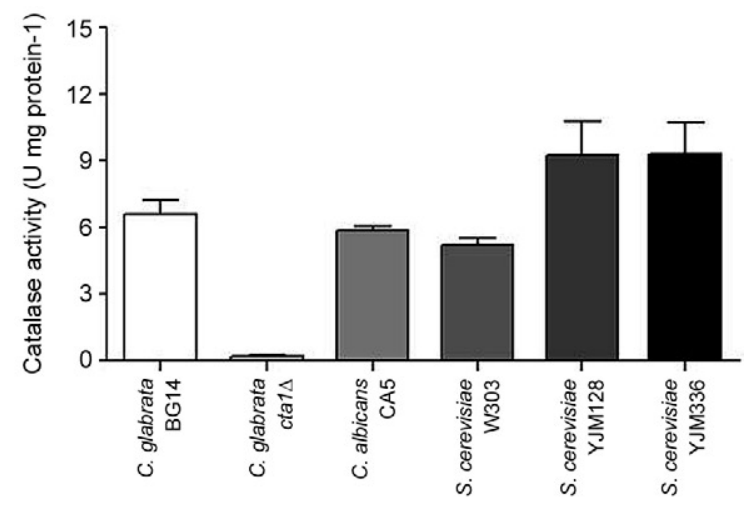

Fig. 4: Candida glabrata, Candida albicans and Saccharomyces cerevisiae catalase activity. C. glabrata strains BG14 and CGM295 (cta1D), C. albicans strain CA5, S. cerevisiae strains W303, CIs YJM128 and YJM336 were grown for $48 \mathrm{~h}$ (stationary phase) in YPD containing $2 \%$ glucose and cell extracts were prepared. The catalase activity was normalized to total protein from the lysate and expressed as units per mg of protein. One unit is defined as the amount of catalase required for degradation of $1.0 \mathrm{\mu mol} \mathrm{H}_{2} \mathrm{O}_{2}$ per min. Data is presented as the mean \pm S.D. of four independent experiments.

catalase. In SP the resistance to menadione is increased and there is no difference in resistance between BG14 and the strain lacking the catalase (cta1D) (Fig. 1B). However, the increased resistance in SP is not surprising. It has previously been shown that SP cells of different pathogens are more resistant to different stresses including oxidative stress (Cyrne et al. 2003). Interest- ingly, Ctal is dispensable in SP and this suggests that other genes are compensating for the lack of Cta1. However, this phenomenon appears to occur only in SP. In LP these genes may be silent. Other elements in SP that could be playing important roles in detoxifying oxidative stress are superoxide dismutases, glutathione, glutathione peroxidases and thioredoxins (Jamieson 1992, Jamieson et al. 1994, Stephen \& Jamieson 1996, Zadzinski et al. 1998). In any case, the regulatory network of the oxidative stress response in SP in C. glabrata is an important aspect that requires further studies.

We compared the resistance to menadione in SP between $C$. glabrata and the closely related non-pathogenic yeast $S$. cerevisiae and the distantly related opportunistic fungal pathogen C. albicans. As shown in Fig. 2, C. albicans strain C.a.CA5 is more resistant to menadione than C. glabrata and S.c.W303 is even more sensitive. The enzymatic removal of superoxide ions is carried out by the enzyme superoxide dismutase. Thus, the resistance to menadione shown by $C$. albicans could be explained, in part, by the fact that $C$. albicans has six superoxide dismutases genes, SOD1, SOD2, SOD3, SOD4, SOD5 and SOD6. It has been shown that C.a.SOD1, C.a.SOD2 and C.a.SOD3 are important for protection in LP and SP and that C.a.SOD1 is important for virulence (Lamarre et al. 2001, Hwang et al. 2002, Martchenko et al. 2004). On the other hand, C. glabrata possesses only two superoxide dismutase genes, C.g.SOD1 and C.g.SOD2, which are orthologs of S. cerevisiae S.c.SOD1 and S.c.SOD2 (Longo et al. 1996, Pereira et al. 2003) and C. albicans C.a.SOD2 and C.a.SOD3. Analysis of the amino acid sequence homology between $C$. glabrata, $S$. cerevisiae and 
C. albicans superoxide dismutases showed that $C$.g. SODs are more closely related to $S . c$.SODs than to C.a.SODs [C.g.Sodlp (CAGL0C04741g) 83\% identical/7\% similar to S.c.Sod1p; C.g.Sod2p (CAGL0E04356g) 70\% identical $/ 11 \%$ similar to S.c.Sod2p, $58 \%$ identical $/ 14 \%$ similar to C.a.Sod2p; $54 \%$ identical $/ 13 \%$ similar to C.a.Sod3p]. Interestingly, both S.c. CIs YJM128 and YJM336 are more resistant to menadione than W303 (Fig. 2) and it has been shown that these CIs are also more resistant to $\mathrm{H}_{2} \mathrm{O}_{2}$ than W303 reference strain (Cuéllar-Cruz et al. 2008). These results support the idea that pathogens need to have a proper response to oxidative damage in order to survive within the host (Clemons et al. 1994, McCullough et al. 1998, Cassone et al. 2003, Munoz et al. 2005). These CIs could have acquired an increased expression/activity of SOD and multiple drug resistant genes and/or reduced permeability.

We investigated the natural resistance of $C$. glabrata to CHP, an organic hydroperoxide. As shown in Fig. 3, C. glabrata is more resistant than C. albicans. S. cerevisiae (W303 and CIs) are almost as resistant as C. glabra$t a$. The oxidative stress generated by CHP is removed in part by glutathione peroxidases and glutaredoxins. One likely explanation of the difference in resistance between C. glabrata and S. cerevisiae and C. albicans could be gene dosage. S. cerevisiae possesses two phospholipid hydroperoxide glutathione peroxidase genes, GPX1 and GPX2, eight glutaredoxin genes, GRX 1-8. C. glabrata possesses five glutaredoxin genes, GRXI -5 and four glutathione peroxidase genes, GPX1-4. Whereas C. albicans possesses only three glutaredoxin genes, GRX1-3.

Analysis of the catalase activity (Fig. 4) showed almost no difference between C. glabrata, $C$. albicans and $S$. cerevisiae, whereas $C$. glabrata has been shown to be more resistant to $\mathrm{H}_{2} \mathrm{O}_{2}$ than C. albicans and S. cerevisiae (Cuéllar-Cruz et al. 2008). This result indicates that there must be additional elements in $C$. glabrata that determine the increased resistance. Interestingly, S. cerevisiae CIs showed increased catalase activity. This result suggests that $S$. cerevisiae CIs could have acquired a more robust response to oxidative stress in order to evade the attack and elimination by phagocytic cells. It would be interesting to determine if Ctal expression is upregulated and/or the activity of Ctal is increased in S. cerevisiae CIs.

Currently, we are making knockout mutants of $C$. glabrata SOD, GPX and GRX genes in order to evaluate their role in the response to menadione and CHP. Furthermore, we are determining whether expression of $S$. cerevisiae Ctal is modified in CIs.

\section{REFERENCES}

Aebi H 1984. Catalase in vitro. Method Enzymol 105: 121-126.

Ausubel F, Brent R, Kingston RE, Moore DD, Seidman JG, Smith JA, Struhl K 2001. Current Protocols in Molecular Biology, John Wiley \& Sons, New York, 670 pp.

Bradford MA 1976. Rapid and sensitive method for microgram quantities of protein utilizing the principle of protein-dye binding. Ann Biochem 72: 248-254.

Cassone M, Serra P, Mondello F, Girolamo A, Scafetti S, Pistella E, Venditti M 2003. Outbreak of Saccharomyces cerevisiae subtype boulardii fungemia in patients neighboring those treated with a probiotic preparation of the organism. J Clin Microbiol 41: $5340-5343$.

Chen KH, Miyazaki T, Tsai HF, Bennett JE 2007. The bZip transcription factor Cgaplp is involved in multidrug resistance and required for activation of multidrug transporter gene CgFLR1 in Candida glabrata. Gene 386: 63-72.

Clemons KV, McCusker JH, Davis RW, Stevens DA 1994. Comparative pathogenesis of clinical and nonclinical isolates of Saccharomyces cerevisiae. J Infect Dis 169: 859-867.

Cormack BP, Falkow S 1999. Efficient homologous and illegitimate recombination in the opportunistic yeast pathogen Candida glabrata. Genetics 151: 979-987.

Cuéllar-Cruz M, Briones-Martin-del-Campo M, Canas-Villamar I, Montalvo-Arredondo J, Riego-Ruiz L, Castano I, De Las Penas A 2008. High resistance to oxidative stress in the fungal pathogen Candida glabrata is mediated by a single catalase, Ctalp, and is controlled by the transcription factors Yap1p, Skn7p, Msn2p, and Msn4p. Eukaryot Cell 7: 814-825.

Cyrne L, Martins L, Fernandes L, Marinho HS 2003. Regulation of antioxidant enzymes gene expression in the yeast Saccharomyces cerevisiae during stationary phase. Free Radic Biol Med 34: 385-393.

Fidel PL, Jr Cutright, JL Tait L, Sobel JD 1996. A murine model of Candida glabrata vaginitis. J Infect Dis 173: 425-431.

Gonzalez-Parraga P, Hernandez JA, Arguelles JC 2003. Role of antioxidant enzymatic defences against oxidative stress $\mathrm{H}_{2} \mathrm{O}_{2}$ and the acquisition of oxidative tolerance in Candida albicans. Yeast 20: 1161-1169.

Hwang CS, Rhie GE, Oh JH, Huh WK, Yim HS, Kang SO 2002. Copper- and zinc-containing superoxide dismutase $\mathrm{Cu} / \mathrm{ZnSOD}$ is required for the protection of Candida albicans against oxidative stresses and the expression of its full virulence. Microbiology 148: 3705-3713.

Jamieson DJ 1992. Saccharomyces cerevisiae has distinct adaptive responses to both hydrogen peroxide and menadione. J Bacteriol 174: 6678-6681.

Jamieson DJ, Rivers SL, Stephen DW 1994. Analysis of Saccharomyces cerevisiae proteins induced by peroxide and superoxide stress. Microbiology 140: 3277-3283.

Kaur R, Domergue R, Zupancic ML Cormack BP 2005. A yeast by any other name: Candida glabrata and its interaction with the host. Curr Opin Microbiol 8: 378-384.

Kaur R, Ma B, Cormack BP 2007. A family of glycosylphosphatidylinositol-linked aspartyl proteases is required for virulence of Candida glabrata. Proc Natl Acad Sci USA 104: 7628-7633.

Lamarre C, LeMay JD, Deslauriers N, Bourbonnais Y 2001. Candida albicans expresses an unusual cytoplasmic manganese-containing superoxide dismutase $S O D 3$ gene product upon the entry and during the stationary phase. J Biol Chem 276: 43784-43791.

Longo VD, Gralla EB, Valentine JS 1996. Superoxide dismutase activity is essential for stationary phase survival in Saccharomyces cerevisiae - Mitochondrial production of toxic oxygen species in vivo. J Biol Chem 271: 12275-12280.

Mansour MK, Levitz SM 2002. Interactions of fungi with phagocytes. Curr Opin Microbiol 5: 359-365.

Martchenko M, Alarco AM, Harcus D, Whiteway M 2004. Superoxide dismutases in Candida albicans: transcriptional regulation and functional characterization of the hyphal-induced SOD5 gene. Mol Biol Cell 15: 456-467. 
McCullough MJ, Clemons KV, Farina C, McCusker JH, Stevens DA 1998. Epidemiological investigation of vaginal Saccharomyces cerevisiae isolates by a genotypic method. J Clin Microbiol 36: 557-562.

McDonald JP, Levine AS, Woodgate R 1997. The Saccharomyces cerevisiae RAD30 gene, a homologue of Escherichia coli dinB and umuC, is DNA damage inducible and functions in a novel errorfree postreplication repair mechanism. Genetics 147: 1557-1568.

Munoz P, Bouza E, Cuenca-Estrella M, Eiros JM, Perez MJ, SanchezSomolinos M, Rincon C, Hortal J, Pelaez T 2005. Saccharomyces cerevisiae fungemia: an emerging infectious disease. Clin Infect Dis 40: 1625-1634.

Nakagawa CW, Mutoh N, Hayashi Y 1995. Transcriptional regulation of catalase gene in the fission yeast Schizosaccharomyces pombe: molecular cloning of the catalase gene and northern blot analyses of the transcript. J Biochem 118: 109-116.

Osorio H, Carvalho E, del Valle M, Sillero MAG, Moradas-Ferreira P, Sillero A 2003. $\mathrm{H}_{2} \mathrm{O}_{2}$, but not menadione, provokes a decrease in the ATP and an increase in the inosine levels in Saccharomyces cerevisiae - An experimental and theoretical approach. Eur J Biochem 270: 1578-1589.

Pereira MD, Herdeiro RS, Fernandes PN, Eleutherio ECA, Panek AD
2003. Targets of oxidative stress in yeast sod mutants. Biochim Biophys Acta 1620: 245-251.

Roetzer A, Gregori C, Jennings AM, Quintin J, Ferrandon D, Butler G, Kuchler K, Ammerer G, Schuller C 2008. Candida glabrata environmental stress response involves Saccharomyces cerevisiae Msn2/4 orthologous transcription factors. Mol Microbiol 69: $603-620$

Stephen DWS, Jamieson DJ 1996. Glutathione is an important antioxidant molecule in the yeast Saccharomyces cerevisiae. FEMS Microbiol Lett 141: 207-212.

Temple MD, Perrone GG, Dawes IW 2005. Complex cellular responses to reactive oxygen species. Trends Cell Biol 15: 319-326.

Thorpe GW, Fong CS, Alic N, Higgins VJ, Dawes IW 2004. Cells have distinct mechanisms to maintain protection against different reactive oxygen species: oxidative-stress-response genes. Proc Natl Acad Sci USA 101: 6564-6569.

Trick WE, Fridkin SK, Edwards JR, Hajjeh RA, Gaynes RP 2002. Secular trend of hospital-acquired candidemia among intensive care unit patients in the United States during 1989-1999. Clin Infect Dis 35: 627-630.

Zadzinski R, Fortuniak A, Bilinski T, Grey M, Bartosz G 1998. Menadione toxicity in Saccharomyces cerevisiae cells: activation by conjugation with glutathione. Biochem Mol Biol Int 44: 747-759. 